\title{
Experimental studies on transient beam loading effects in the presence of a superconducting third harmonic cavity
}

\author{
Giuseppe Penco and Michele Svandrlik \\ Sincrotrone Trieste, Strada Statale 14-km 163,5 in AREA Science Park, 34012 Basovizza, Trieste, Italy
}

(Received 7 December 2005; published 10 April 2006)

\begin{abstract}
In the framework of the SUPER-3HC project, an idle superconducting third harmonic cavity is installed in the ELETTRA storage ring in order to lengthen the bunch, thus improving the Touschek Lifetime. An improvement of more than a factor three of the beam lifetime is obtained, and the induced Landau damping renders ELETTRA longitudinally stable for the first time. The paper describes the performed studies and the experimental results, showing a strong correlation between the presence of an empty gap in the filling pattern of the ring and the third harmonic cavity induced Landau damping of the longitudinal instabilities, the bunch lengthening modulation along the bunch train and the beam lifetime improvement. Finally, an experimental investigation on the bunch profile overstretching, leading to find a low overstretching threshold below which the beam lifetime continues to increase, is presented.
\end{abstract}

DOI: 10.1103/PhysRevSTAB.9.044401

PACS numbers: 29.27.Bd, 29.20.Lq

\section{INTRODUCTION}

The main challenge of the third generation synchrotron light sources is to increase the beam lifetime. In the lowmedium energy machine, as ELETTRA, the beam lifetime is mainly dominated by large-angle intrabeam scattering, i.e., Touschek scattering. In the past ELETTRA, when operated at $2.0 \mathrm{GeV}$, applied an amplitude stable longitudinal coupled-bunch mode, obtained by a fine tuning of the rf cavities temperature [1]. The longitudinal bunch dilution produced by that mode increased by $30 \%$ the beam lifetime, providing in the meantime a Landau damping of all the transversal instabilities. Recently, an interesting solution extensively discussed in several laboratories has been adopted, consisting of using a higher harmonic rf cavity to lengthen the bunches. In fact, by manipulating the harmonic voltage and the phase in order to flatten the total voltage sampled by the beam, the bunches are lengthened, therefore improving the Touschek lifetime, thanks to the reduced longitudinal bunch charge, without affecting the transverse emittance and thus the radiation brightness. Moreover, the nonlinearity of the rf voltage experienced by the bunches also provides a Landau damping of the longitudinal couple bunch mode (CBM) instabilities, allowing ELETTRA to even operate with longitudinal stability.

During the summer of 2002, an idle superconducting third harmonic cavity (3HC) was installed in the ELETTRA storage ring, in the framework of the SUPER$3 \mathrm{HC}$ project [2], aimed to install two $3 \mathrm{HC}$ cavities, one in the ELETTRA and one in the SLS storage rings. Several theoretical studies carried out in the past decade [3-7] underlined the advantages obtained by using a superconducting (SC) cavity rather than a normal conducting one.

*Electronic address: giuseppe.penco@elettra.trieste.it
Application of this solution is expected to improve the beam lifetime by a factor of 3 .

The SUPER-3HC represents the first superconducting application of a high harmonic rf system in a storage ring, providing not only a relevant improvement in terms of beam lifetime and longitudinal stability but also offering the big opportunity to characterize the beam in a more exhaustive way. Indeed, the very high quality factor $\left(Q_{0}\right)$ of the SC cavity and the associated narrow bandwidth allows tuning of the $3 \mathrm{HC}$ cavity very near to the third harmonic of the beam, without exciting longitudinal instabilities, leading to investigate beam dynamics phenomena (as the overstretching of the bunch profile) still not explored to date.

This paper does not deal in detail with the beam dynamics effects produced by a higher harmonic voltage. Please refer to [7-9] for a complete description, while only a number of basic results shall be recalled here. Using a passive harmonic cavity is a simple solution because an external rf power generator is not required to provide the voltage, which is generated by the beam itself. The power lost by the beam [5] passing through an idle cavity is

$$
P_{b}=V \cdot I_{b} \cos (\psi)=\frac{V^{2}}{2 R_{s}},
$$

where $V=2 R_{s} I_{b} \cos \psi$ is the voltage induced in the cavity by the beam current $I_{b}, R_{s}$ is the cavity shunt impedance, and $\psi$ is the cavity tuning angle, defined as

$$
\tan (\psi)=\frac{2 Q_{0} \cdot \delta \omega}{\omega_{r}}
$$

where $\omega_{r}$ is the resonant frequency of the cavity and $\delta \omega$ is the cavity frequency detuning. If the cavity detuning is large in comparison with the resonance bandwidth as in the superconducting case $\left(\delta \omega \gg \omega_{r} / Q_{0}\right)$, the tuning angle becomes $\psi \sim \pi / 2$, the power given by formula (1) is almost zero, and the voltage generated in the cavity can 
be expressed as

$$
V \sim I_{b} \cdot R / Q \cdot \frac{\omega_{r}}{\delta \omega},
$$

where $R / Q$ is the cavity geometric factor.

The combined voltage from the main and harmonic rf system is given by

$$
V(t)=V_{\mathrm{rf}} \cdot \sin \left(\omega_{r f} t+\phi_{s}\right)+V_{h} \cdot \sin \left[n\left(\omega_{\mathrm{rf}} t+\phi_{h}\right)\right],
$$

where the variable $t$ refers to the time displacement with respect to the synchronous particle, $V_{\mathrm{rf}}$ is the main $\mathrm{rf}$ voltage, $V_{h}$ is the harmonic voltage, $\phi_{s}$ and $\phi_{h}$ are the stable phases between the bunch and the main and harmonic voltage, and $n$ is the $\mathrm{rf}$ frequency harmonic. The longitudinal bunch distribution can be shaped by varying the relative amplitude and phase of the harmonic voltage and thus the rf potential, that is given by

$$
U(\phi)=\frac{1}{V_{\mathrm{rf}} \cos \left(\phi_{s}\right)} \int_{0}^{\phi}\left(V(\phi)-U_{0}\right),
$$

where $\phi=\omega_{\mathrm{rf}} t$ and $U_{0}$ is the energy lost per turn.

By varying the harmonic phase $\phi_{h}$ and the voltage $V_{h}$, the harmonic cavity may operate in lengthening or in shortening mode according to the voltage seen by the beam, which may have a correspondingly flattened or increased slope. When the beam current decays, the cavity should be tuned closer to the harmonic to keep the harmonic voltage constant, and this could become an issue for low current. Thus an input coupler, currently disconnected, is already installed on the cavity, allowing for a possible future active mode operation. In lengthening mode, in order to maximize the bunch length without generating multiple stable points in the rf bucket, the phase and amplitude of the harmonic voltage are adjusted so that both the first and second derivatives of the total voltage vanish near the synchronous phase [8]. This leads to an optimum setting of the parameters $\phi_{h}$ and $V_{h}$ :

$$
\begin{aligned}
& k_{\mathrm{opt}}=\frac{V_{h, \mathrm{opt}}}{V_{\mathrm{rf}}}=\sqrt{\frac{1}{n^{2}}-\frac{\left(U_{0} / V_{\mathrm{rf}}\right)^{2}}{n^{2}-1},} \\
& \sin \left(n \phi_{h, \mathrm{opt}}\right)=\frac{-U_{0}}{V_{h, \mathrm{opt}}\left(n^{2}-1\right)} .
\end{aligned}
$$

By calculating formula (6) for the machine parameters of the ELETTRA storage ring (Table I) the optimum voltage that should be provided with a third harmonic cavity turns out to be approximately $553 \mathrm{kV}\left(k_{\mathrm{opt}} \sim\right.$ 0.329 ). At $2.0 \mathrm{GeV}$, in order to obtain this voltage at $320 \mathrm{~mA}$, calculations based on formula (3) show that the cavity detuning should be about $+80 \mathrm{kHz}$, assuming $R / Q=88 \Omega$.

An extensive effort to measure the bunch lengthening effect and the relative implication on the beam lifetime and
TABLE I. ELETTRA machine parameters.

\begin{tabular}{lc}
\hline \multicolumn{1}{c}{ Beam energy [GeV] } & 2.0 \\
\hline Beam revolution frequency [MHz] & 1.1566 \\
Harmonic number & 432 \\
Energy lost per turn without ID's [keV] & 255.7 \\
Max. energy lost per turn with all ID's [keV] & 315 \\
Injected current [mA] & 320 \\
Energy spread (rms)\% & 0.08 \\
Momentum compaction $\alpha$ & $1.6 \cdot 10^{-3}$ \\
Nominal lifetime [hours] (before 3HC) & 8 \\
Nominal rms bunch length (mm) & 5.4 \\
Main rf frequency [MHz] & 499.654 \\
Main rf peak voltage [kV] & 1680 \\
Third harmonic frequency [MHz] & 1498.962 \\
\hline \hline
\end{tabular}

beam stability has been carried out at ELETTRA. The ELETTRA storage ring was operated in the past with a $10 \%$ empty gap in the electron filling pattern in order to clean trapped ions. The temporal asymmetry in the bunch train induces a modulation of the harmonic voltage sampled by each bunch, with a consequent modulation of the synchronous phase along the bunch train, as described in $[9-11]$. Thus the optimum conditions reported in the formulas (6) and (7) are not respected for all the bunches: in particular, the phase experienced by the head and the tail bunches in the train is significantly different from the optimum one. The effect of this phase modulation on the amplitude of the CBMs and on the Landau damping, on the bunch lengthening, and on the beam lifetime has been studied as a function of the $3 \mathrm{HC}$ detuning, for different machine fillings, from uniform filling (432 filled buckets) to $70 \%$ filling (303 filled buckets and 129 empty buckets, i.e., with a gap of $258 \mathrm{~ns}$ ).

\section{LANDAU DAMPING}

When the harmonic phase is adjusted in lengthening mode, the nonlinearity of the rf voltage leads to a solution of the equation of motion with a synchrotron frequency dependence on the amplitude. This induces a spread in synchrotron frequencies within bunches, that helps in damping longitudinal coupled-bunch instabilities through the Landau mechanism [11]. In the presence of an empty gap in the bunch train, as discussed below, the effect of the third harmonic cavity may be reduced, as well as eventually the contribution to the Landau damping. Nevertheless the empty gap itself introduces a nonlinearity that induces a synchrotron frequency spread [11]. In this framework we have measured the amplitude of the CBMs during the tuning of $3 \mathrm{HC}$ for different fractional fillings, in order to study how these complementary contributions are balanced.

When $3 \mathrm{HC}$ is tuned far away from the 3rd harmonic, the excited CBMs pattern is strongly dependent from the frac- 
tional filling. As expected, the amplitude of the CBMs becomes smaller as the empty gap is increased. Particularly, for a filling of 70\% (303 filled buckets and 129 empty buckets) at $315 \mathrm{~mA}, 2.0 \mathrm{GeV}$, the longitudinal spectrum of the beam only shows one mode with an amplitude larger than $1^{\circ}$, even if $3 \mathrm{HC}$ is detuned (see Fig. 1). This is because of the large damping contribution coming from the large empty gap. Interestingly enough, however, obtaining a completely stable situation by activating $3 \mathrm{HC}$ under these conditions is much more difficult. In fact, the beam is stabilized only at a frequency detuning of $+59 \mathrm{kHz}$. Here the harmonic voltage $V_{h \text {,th }}$, calculated for uniform filling reaches $+700 \mathrm{kV}$, that is much more than the optimum value of $560 \mathrm{kV}$. This confirms that for large empty gaps the effect of the phase modulation along the bunch train is considerably amplified and the formula (3) is no more applicable because each bunch experiences a different harmonic voltage and phase. When tuning $3 \mathrm{HC}$, the smaller the empty gap, the faster the increase of the Landau damping contribution coming from the harmonic voltage. Figure 2 shows the CBMs amplitude for a $80 \%$ filling pattern as a function of the $3 \mathrm{HC}$ detuning. In this case for large $3 \mathrm{HC}$ detuning the Landau damping provided by the empty gap is lower than in the previous case and three modes have an amplitude of a few degrees. Otherwise the $3 \mathrm{HC}$ tuning is much more effective, and all the longitudinal modes are damped already at a frequency detuning of $+90 \mathrm{kHz}$.

The Landau damping provided by $3 \mathrm{HC}$ tuning is more amplified in the standard filling of $90 \%$ (see Fig. 3): a progressive damping of the CBMs has been registered, and its amplitude decreasing from $20^{\circ}$ to $10^{\circ}$ at a $3 \mathrm{HC}$ detuning of $+114 \mathrm{kHz}$. Just below this detuning (at $+104 \mathrm{kHz}$ ) all CBMs are suddenly suppressed. The harmonic voltage $V_{h, \text { th }}$ generated in the cavity at this frequency detuning,

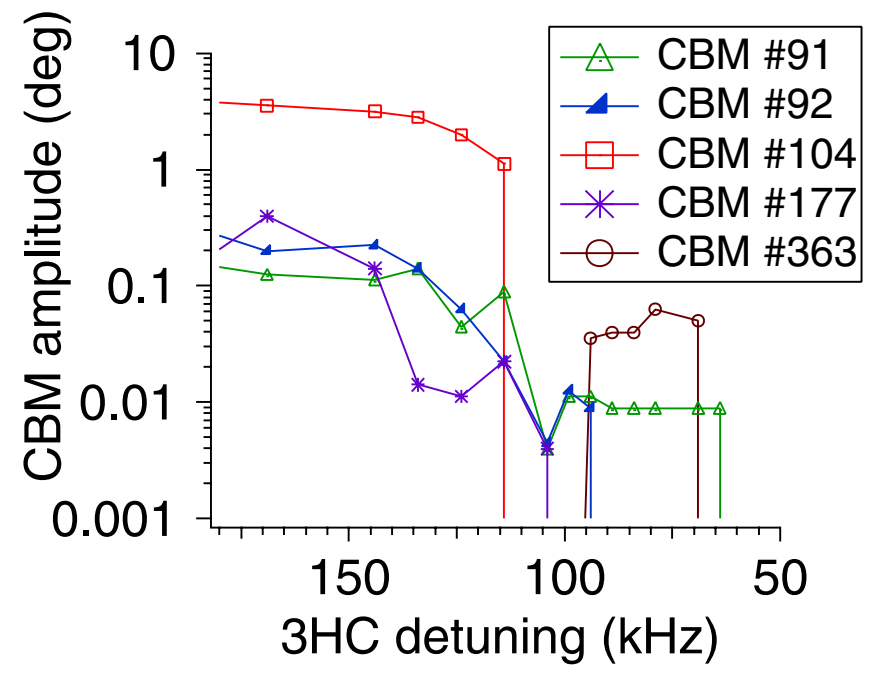

FIG. 1. (Color) CBMs amplitude versus $3 \mathrm{HC}$ tuning for a $70 \%$ filling of the ring; $I_{\text {beam }}=315 \mathrm{~mA}, E=2.0 \mathrm{GeV}$.

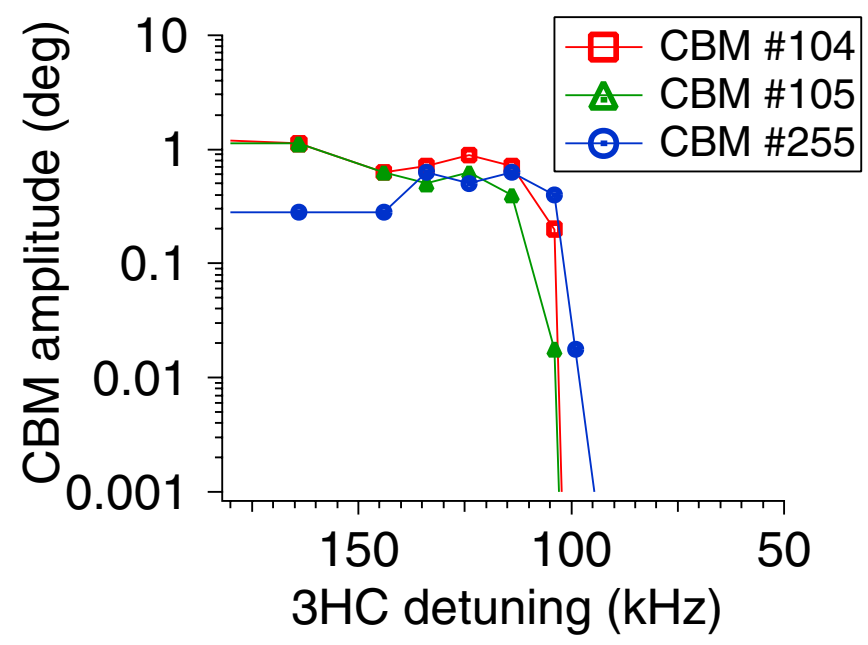

FIG. 2. (Color) CBMs amplitude versus $3 \mathrm{HC}$ tuning for a $80 \%$ filling of the ring; $I_{\text {beam }}=315 \mathrm{~mA}, E=2.0 \mathrm{GeV}$.

calculated from (3), results to be $400 \mathrm{kV}$, which is about $70 \%$ of the optimum voltage.

In the uniform filling case at large detuning of $3 \mathrm{HC}$ many CBMs are present at large amplitude $\left(\sim 25^{\circ}\right)$ because of the absence of Landau damping sources (Fig. 4). Tuning $3 \mathrm{HC}$, its interaction with the beam is much more effective than in the presence of a gap. All modes but the CBM 110 are abruptly killed already at $+104 \mathrm{kHz}$. Unlike the case of $90 \%$ filling, in order to also damp the CBM 110 and definitely stabilize the beam, further detuning $3 \mathrm{HC}$ to $+94 \mathrm{kHz}$ is necessary, where $V_{h, \text { th }}$ becomes equal to $440 \mathrm{kV}$. For uniform filling, the Landau damping is provided only by $3 \mathrm{HC}$, while in the case of fractional filling the gap too contributes to the damping, while not allowing a completely stable beam, unless $3 \mathrm{HC}$ is activated.

The conclusion is that, for the ELETTRA storage ring, the Landau damping contribution coming from the activation of $3 \mathrm{HC}$ is stronger than the presence of an empty gap:

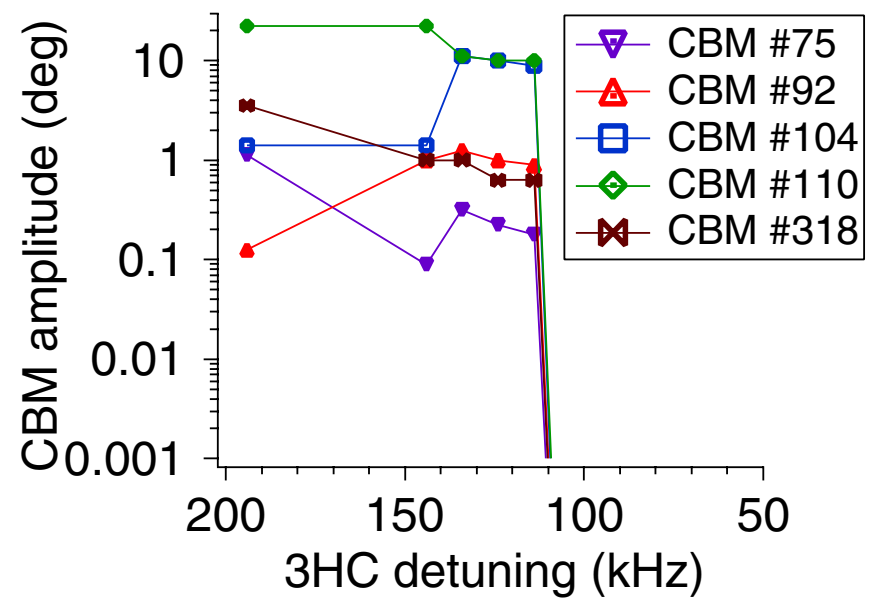

FIG. 3. (Color) CBMs amplitude versus $3 \mathrm{HC}$ tuning for a $90 \%$ filling of the ring; $I_{\text {beam }}=315 \mathrm{~mA}, E=2.0 \mathrm{GeV}$. 


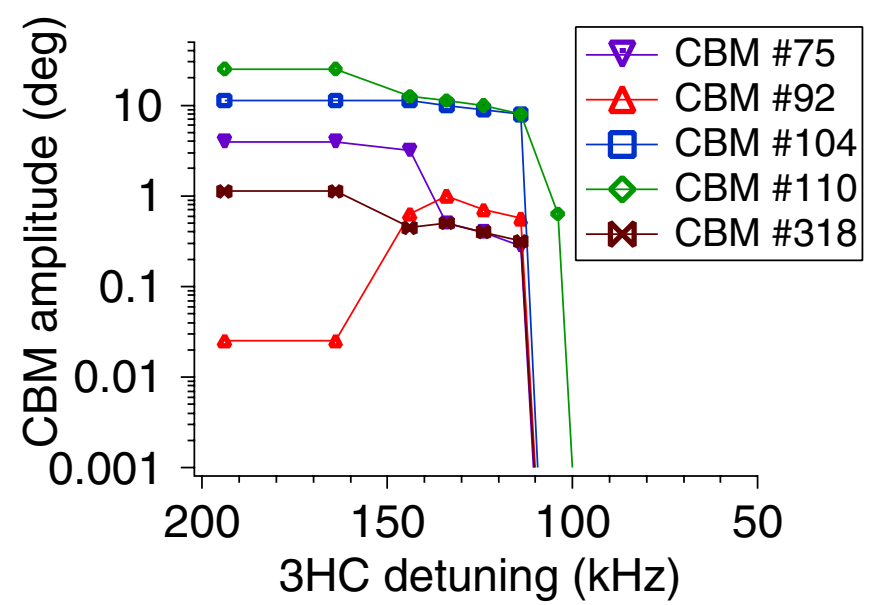

FIG. 4. (Color) CBMs amplitude versus $3 \mathrm{HC}$ tuning for a uniform filling of the ring; $I_{\text {beam }}=315 \mathrm{~mA}, E=2.0 \mathrm{GeV}$.

even in uniform filling, starting with CBMs excited with amplitudes around $25^{\circ}$, by tuning $3 \mathrm{HC}$ it is possible to suppress all longitudinal coupled-bunch instabilities.

\section{PHASE SHIFT}

This paragraph reports the measurements of the phase shift induced by the empty gap for different fractional fillings of the storage ring. By using the streak camera equipment [12,13] a large number of images has been acquired when tuning $3 \mathrm{HC}$, which allowed measurement of the phase modulation along the bunch train. The image data consist of an array of $736 \times 572$ points, which correspond, in our experimental setup, to a window of $1350 \mathrm{~ns}$ (vertically) $\times 872 \mathrm{ps}$ (horizontally). Fitting each data row with Gaussian curves, the position of the peak center and the rms longitudinal dimension of each bunch were obtained. Remarkably, for large bunch elongation the

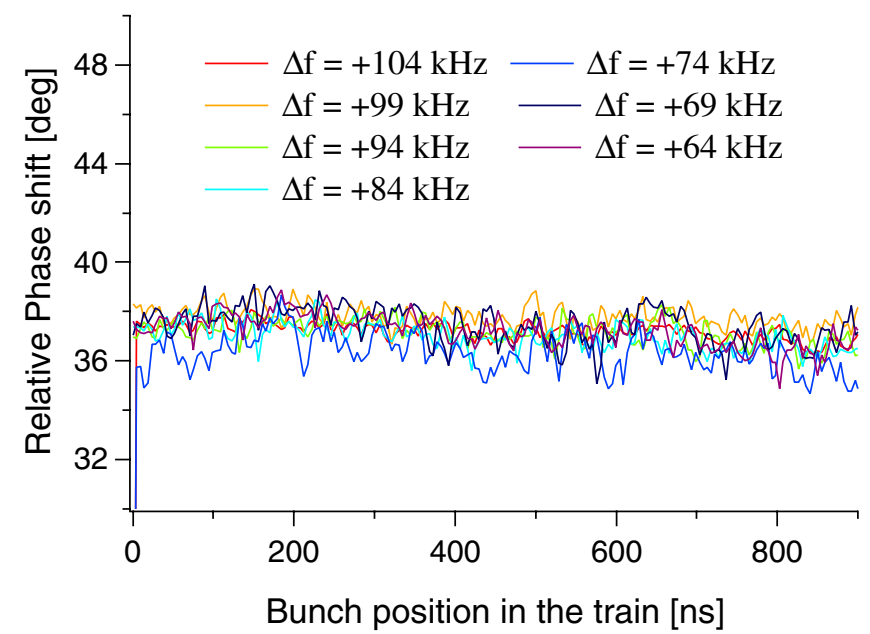

FIG. 5. (Color) Relative stable phase along the bunch train vs the $3 \mathrm{HC}$ detuning, for uniform filling; $I_{\text {beam }}=315 \mathrm{~mA}, E=$ $2.0 \mathrm{GeV}$.

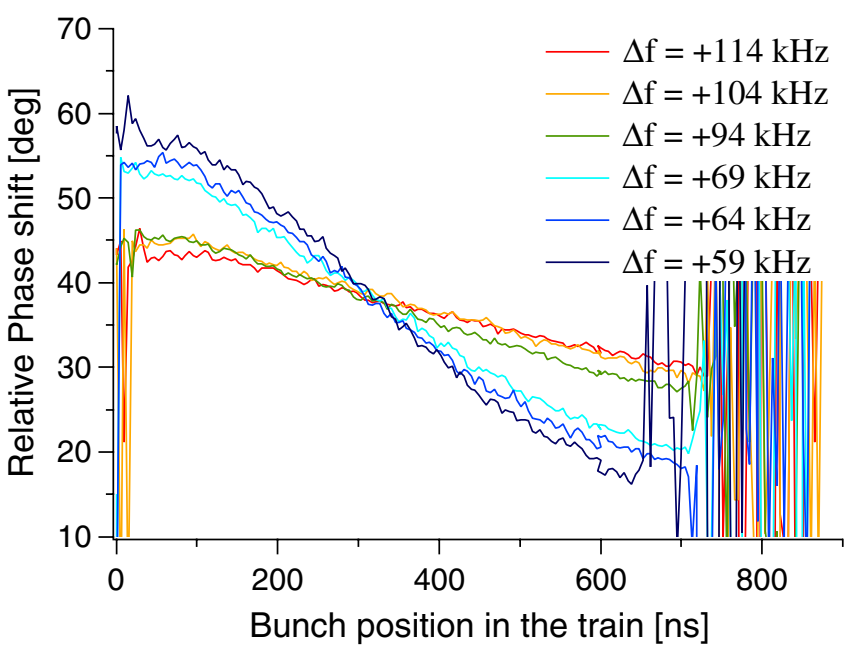

FIG. 6. (Color) Relative stable phase along the bunch train vs the $3 \mathrm{HC}$ detuning, for a $80 \%$ filling; $I_{\text {beam }}=315 \mathrm{~mA}, E=$ $2.0 \mathrm{GeV}$.

bunch profile is no longer Gaussian, so the rms bunch length value has been always considered rather than the rms length of the Gaussian fit. First of all, the measurement in Fig. 5 confirms that, in the absence of an empty gap (uniform filling), the temporal symmetry leads all bunches to the same stable phase, independently from the $3 \mathrm{HC}$ frequency detuning. Also in the case of a filling of $80 \%$, the transient beam loading confirms to induce a relevant synchronous phase modulation along the bunch train; the effect is amplified proportionally to the increasing of the harmonic voltage as shown in Fig. 6: for a 3HC detuning of $+59 \mathrm{kHz}$, the phase difference between the head and the tail of the bunch train is equal to $40^{\circ}$.

These measurements have been repeated for different fillings, and the phase difference between the head and the tail of the bunch train versus the $3 \mathrm{HC}$ frequency detuning for each fractional filling is reported in Fig. 7.

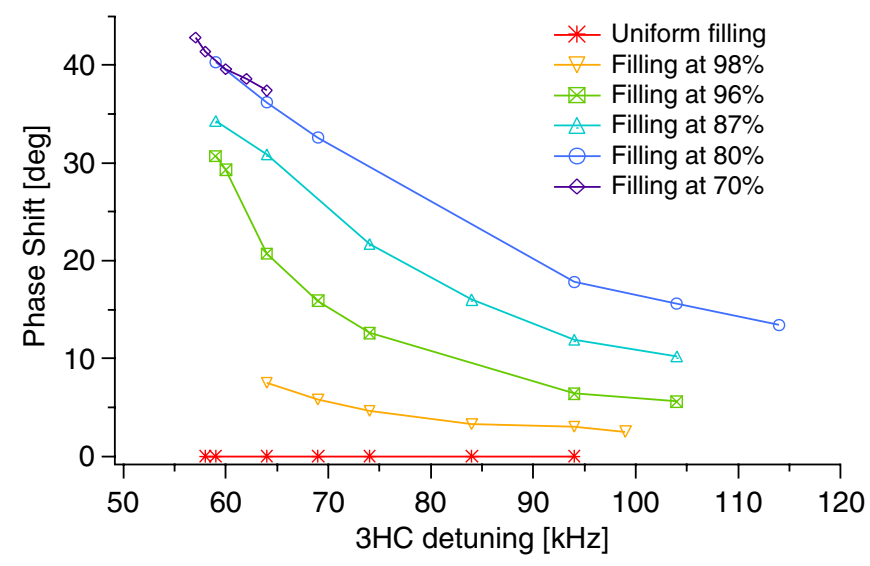

FIG. 7. (Color) Phase difference between the head and the tail of the bunch train vs the $3 \mathrm{HC}$ detuning, for several fractional fillings; $I_{\text {beam }}=315 \mathrm{~mA}, E=2.0 \mathrm{GeV}$. 
The head-tail phase difference is obtained by linear fitting the streak camera data relative to the central position of the bunches along the train. This measurement confirms the theoretical expectation that the larger the empty gap, the larger the phase modulation along the bunches; for a $30 \%$ empty gap the difference in phase between the head and the tail reaches $43^{\circ}$. The noise between the central position of subsequent bunches, obtained as the peak position of the Gaussian fitting, is not negligible in the case of small empty gap. In particular in the uniform filling case the linear fitting provides head-tail phase differences lower than $1^{\circ}$, while the noise of the acquired data has an amplitude of about $2^{\circ}$. This confirms that for the uniform filling case no phase shift is observed. For large 3HC detuning the experimental curves in Fig. 7 asymptotically tend to a value which is not zero. The "residual" phase modulation is determined by both to the beam voltage generated in the $3 \mathrm{HC}$, which is not negligible even when tuned between two revolution frequencies, and to the contribution of the main rf system. For example, in the case of a $80 \%$ filling, a beam current of $320 \mathrm{~mA}$ in $3 \mathrm{HC}$ (tuned at $+578 \mathrm{kHz}$, that is half of the revolution frequency equal to $1156.6 \mathrm{kHz}$ ) generates a harmonic voltage of about $85 \mathrm{kV}$, inducing a total phase shift of about $5^{\circ}$ with the main $\mathrm{rf}$ system.

\section{BUNCH LENGTHENING}

The consequence of the synchronous phase modulation provided by the transient beam loading due to the asymmetric filling, described in the previous paragraph, is a modulation pattern of the harmonic voltage experienced by each bunch, not only in amplitude but also in phase. This modulation leads to the fact that not all bunches are lengthened uniformly. In order to understand this effect, a tracking code developed in collaboration between facilities at ESRF and ALS $[9,10]$ has been used to calculate the steady-state harmonic voltage and the harmonic phase experienced by each bunch, for an $80 \%$ filling and a $3 \mathrm{HC}$ detuning of $+144 \mathrm{kHz}$. The results are plotted in Fig. 8. A detailed analysis of the obtained results clearly shows that bunches in the head of the train experience the maximum harmonic voltage but with a harmonic phase shift of about $15^{\circ}$ with respect to the optimum condition $\left(\sim 90^{\circ}\right)$. Otherwise the bunches in the middle have the right harmonic phase but the smaller harmonic voltage, which is reduced by $10 \%$ in the case shown in Fig. 8 . The particle distribution within the bunch and its peak position (synchronous phase) are very sensitive to small variation in the harmonic voltage and phase, mainly because of the reduction of the longitudinal focusing, due to the rf voltage flattening. Hence the voltage transient induced by the gap is amplified. Thus for $3 \mathrm{HC}$ detuning near the optimum condition, as shown in Fig. 9 in the 90\% filling case, the harmonic voltage experienced by the tail and the head of the bunch train is near the optimum $(513 \mathrm{kV})$ but is out of

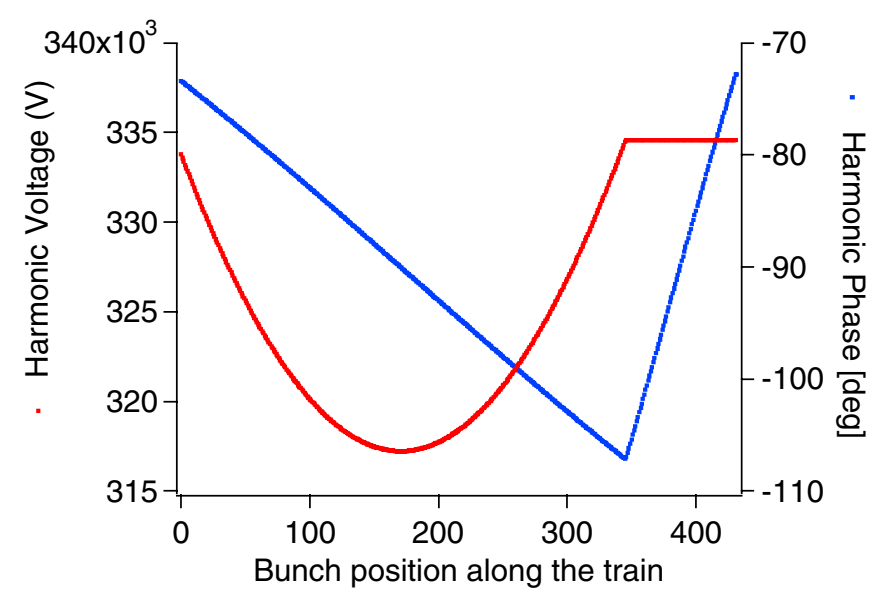

FIG. 8. (Color) ESRF code results of the harmonic voltage (red) and phase (blue) modulation along the bunch train after the transient beam loading, obtained for a beam current of $315 \mathrm{~mA}$, a $80 \%$ filling and a $3 \mathrm{HC}$ detuning of $+144 \mathrm{kHz}$.

phase, and the lengthening effect of these bunches is dramatically reduced. The bunches in the middle are just in phase but they sample a harmonic voltage of only $480 \mathrm{kV}$, so their lengthening is about $90 \%$ of the optimum.

As for the phase shift several experiments have been carried out to measure the bunch length of each bunch in function of the $3 \mathrm{HC}$ detuning for different fractional fillings. In order to have a reference bunch length, the bunch length has been initially measured at "almost" zero current $(I=15 \mathrm{~mA}$ at $2.0 \mathrm{GeV})$, when $3 \mathrm{HC}$ is parked far away from the third harmonic. Fitting the data with a Gaussian distribution we obtained a rms bunch length of $22.6 \mathrm{ps}$, which is quite in agreement with the nominal value of 18 ps.

The analysis at different fractional fillings, performed as described in previous section, allow for plotting the bunch length modulation along the train for fillings varying from

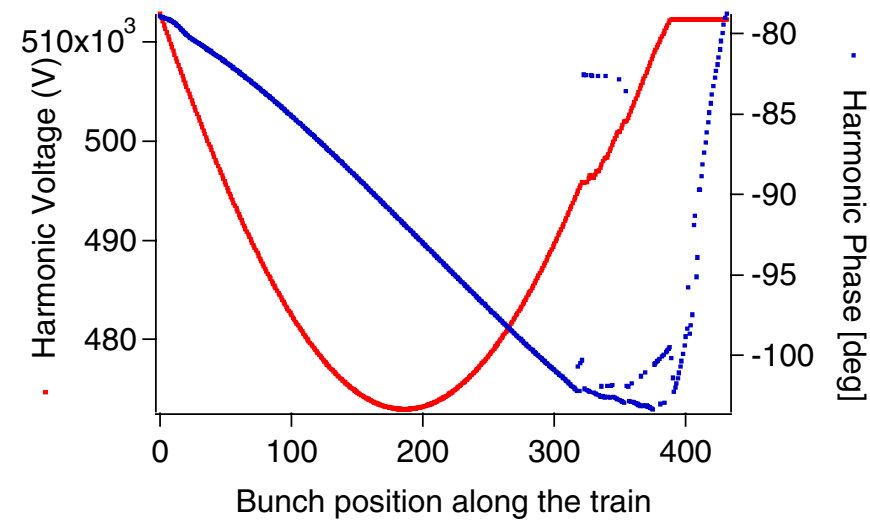

FIG. 9. (Color) ESRF code results of the harmonic voltage (red) and phase (blue) modulation along the bunch train after the transient beam loading, obtained for a beam current of $315 \mathrm{~mA}$, a $90 \%$ filling and a $3 \mathrm{HC}$ detuning of $+81 \mathrm{kHz}$. 
$70 \%$ to $100 \%$. As an example Fig. 10 reports the $90 \%$ filling case, corresponding to the standard fractional filling used at ELETTRA before 3HC was installed, in comparison with the uniform filling case shown in Fig. 11.

In the case of nonuniform filling the bunches in the middle of the train are lengthened proportionally to the harmonic voltage increment, caused by the $3 \mathrm{HC}$ detuning, while the head and the tail of the bunch train behave as if they would suffer a saturation effect: being out of phase with respect to the harmonic voltage, they do not experience a flat potential. For small gaps the transient beam loading is reduced and a larger portion of the bunch train is lengthened with the increasing of the $3 \mathrm{HC}$ voltage. Finally for a uniform filling (see Fig. 11) all bunches equally experience the same harmonic voltage, so they have the same synchronous phase and thus they are lengthened by the same amount. The $3 \mathrm{HC}$ detuning corresponding to the optimum harmonic voltage necessary to flatten the potential well, in a uniform filling and at $315 \mathrm{~mA}$, is $+75 \mathrm{kHz}$. Under these conditions, theory would have the rms bunch length increased by a factor 3 approximately. Our experimental results have been compared with the theoretical expectation, starting first of all by considering the uniform filling case. The rms bunch length in general is given by

$$
\sigma_{\phi}=\sqrt{\frac{\int_{-\pi}^{+\pi} \overline{\phi^{2} \Psi(\phi) d \phi}}{\int_{-\pi}^{+\pi} \Psi(\phi) d \phi}},
$$

where $\Psi(\phi)$ is the particle distribution, strongly dependent from the rf potential $U(\phi)$, given by (5), as [9]:

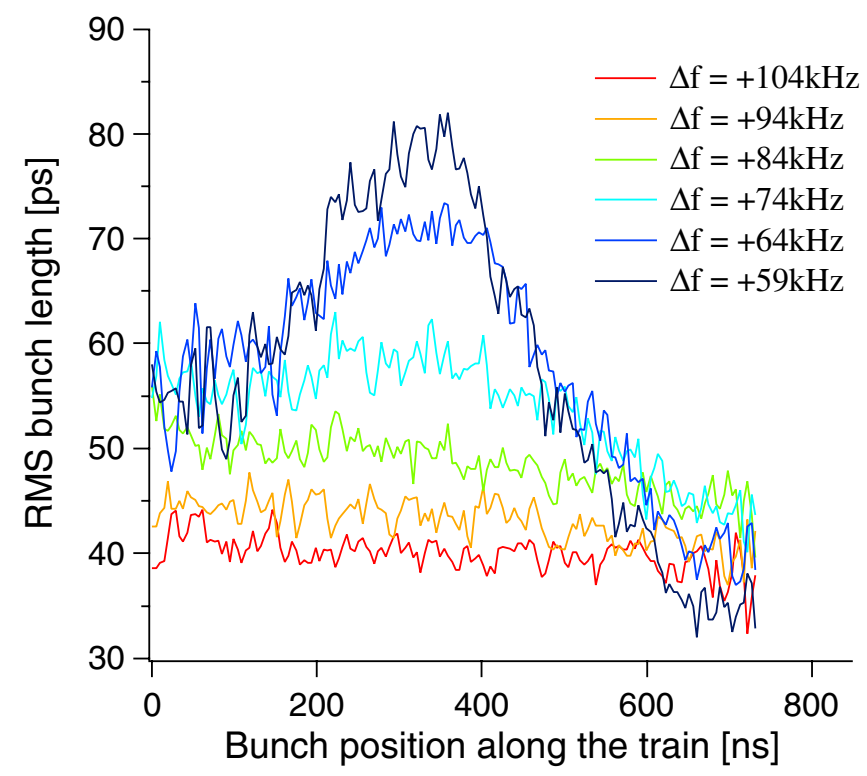

FIG. 10. (Color) rms bunch length along the bunch train for several $3 \mathrm{HC}$ tuning for a filling of $90 \%$; $I_{\text {beam }}=315 \mathrm{~mA}, E=$ $2.0 \mathrm{GeV}$.

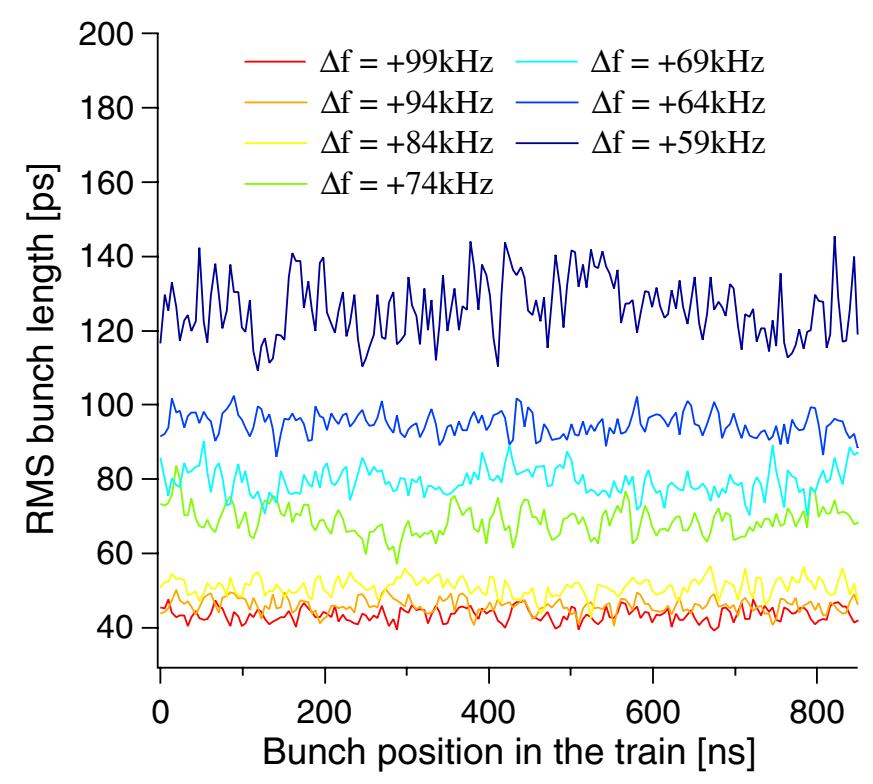

FIG. 11. (Color) rms bunch length along the bunch train for several 3HC tuning for uniform filling; $I_{\text {beam }}=315 \mathrm{~mA}, E=$ $2.0 \mathrm{GeV}$.

$$
\Psi(\phi)=\bar{\Psi} \exp \left(-\frac{U(\phi)}{\alpha^{2} \sigma_{\epsilon}^{2}}\right)
$$

with the normalization constant $\bar{\Psi}$.

In order to perform a reliable comparison experiment/ theory, Fig. 12 reports the plotting of the lengthening factor measured in two experiments at uniform filling, with respect to the zero current rms bunch length measured previously (22.6 ps), and the theoretical lengthening with respect to the nominal zero current bunch length (18.5 ps), obtained by applying again formula (8) without

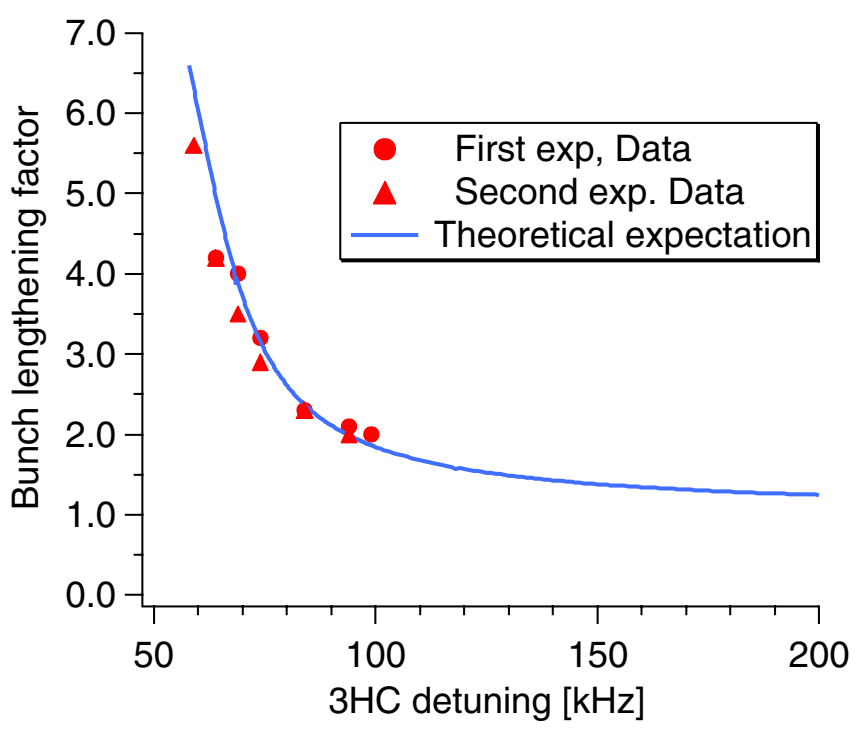

FIG. 12. (Color) Comparison between two set of experimental data, obtained in the same machine condition, and theoretical calculation for a uniform filling pattern, at $315 \mathrm{~mA}, 2.0 \mathrm{GeV}$. 
including the harmonic voltage. Remarkably, independently from the assumed bunch profile, formula (8) provides the rms longitudinal dimension, which corresponds to what has been measured from the streak camera data. The good agreement between the theoretical expectation and the results of our experiments shown in Fig. 12 demonstrates the accuracy of the analytical approach to the bunch lengthening calculation in case of uniform filling. Moreover in Fig. 12 the comparison experiments/theory is extended below the optimum $3 \mathrm{HC}$ detuning of $+75 \mathrm{kHz}$ : in this region the potential well is distorted so that the particles distribution within each bunch is split in two semistable regions (bunch overstretching). Under these conditions, the rms longitudinal distribution, defined in (8), may achieve large value, as measured in Fig. 11 and as the theory predicts. Particularly, for a $3 \mathrm{HC}$ detuning of $+64 \mathrm{kHz}$, a rms bunch length of about $100 \mathrm{ps}$ has been measured, which is almost a factor 5 in comparison with the zero current bunch length previously measured, and reducing the detuning further (at $+59 \mathrm{kHz}$ ), the average rms distribution attains $125 \mathrm{ps}$, that is about 5.5 times the zero current bunch length. The overstretching effect is described in detail in the next paragraph.

While it is possible to treat the bunch lengthening in uniform filling case analytically, in the case of fractional fillings it is very difficult to predict the theoretical bunch length with analytical formulas. By using the existing multiparticles tracking codes, as the one developed at the ESRF [10], obtaining a good description of the bunch lengthening effect is indeed possible, even fairly close to the optimum tuning condition (potential well flattening), while at optimum condition or over (overstretching) the code does not always converge to reliable results.

The bunch lengthening modulation along the bunch train in different fractional filling configuration from $70 \%$ to $100 \%$ has been measured. In order to give an estimation of the effectiveness of $3 \mathrm{HC}$ in the bunch lengthening at different fillings, the rms bunch length along the train for all our experiments has been averaged. The results are plotted in Fig. 13. For $20 \%$ or $30 \%$ empty gaps, the bunch lengthening effect averaged along the train saturates after an initial increment: an average rms bunch length of, respectively, 50 and $45 \mathrm{ps}$ is obtained, when $3 \mathrm{HC}$ is detuned at $\sim+60 \mathrm{kHz}$. Reducing the gap the global lengthening of bunches increases: for a filling of $96 \%$ the rms bunch length at $+59 \mathrm{kHz}$ reaches approximately $75 \mathrm{ps}$, while in uniform filling it reaches even $125 \mathrm{ps}$. As described in the last paragraph, the effective improvement in the Touschek lifetime coming from the bunch lengthening, being an average effect on the bunch train, is strongly affected in a similar way by the presence of an empty gap.

\section{BUNCH OVERSTRETCHING}

The particle distribution within the bunch strongly depends on the rf voltage, determining the stable region

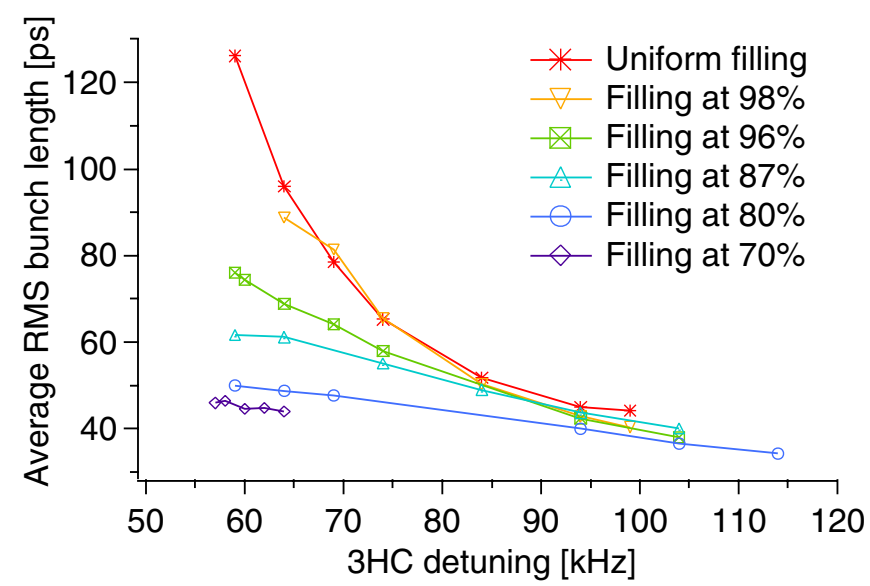

FIG. 13. (Color) Average rms bunch length along the bunch train for several $3 \mathrm{HC}$ tuning; $I_{\text {beam }}=315 \mathrm{~mA}, E=2.0 \mathrm{GeV}$.

towards which all particles with an energy different from the nominal, but still within the energy acceptance, are focused. The rf potential well $U(\phi)$ [formula (5)], if deformed beyond the optimum flat condition, creates two stable points around the synchronous phase and the bunch profile presents a double peak, as shown in Figs. 14 and 15.

First of all the effort has been made to characterize the overstretching effect in the simplest case, that is in a uniform filling pattern, which is finally not affected by the head-tail phase shift described before. Thus the streak camera data relative to subsequent bunches can be superimposed in order to build an average bunch profile. The streak camera data acquired at different $3 \mathrm{HC}$ detuning with the corresponding best fitting curve are reported in Fig. 16. At $+114 \mathrm{kHz}$ [Fig. 16(a)] the bunch profile is still well fitted by a single Gaussian, while at $+74 \mathrm{kHz}$ [Fig. 16(b)], corresponding to a flattened potential, the bunch profile should be flat, so that the sum of 2 Gaussians provides the

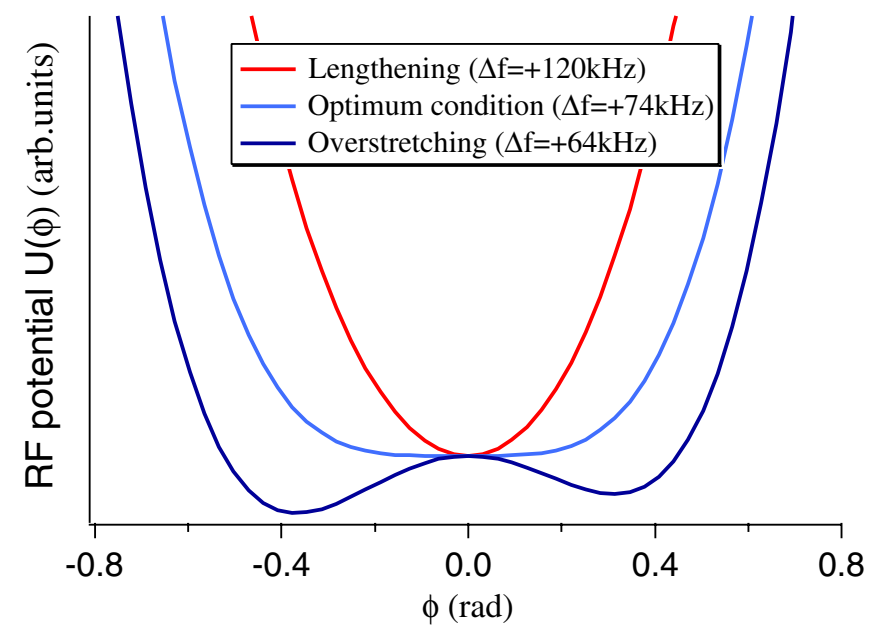

FIG. 14. (Color) Potential well distortion in lengthening mode, at optimum condition (flattened) and in overstretching regime, calculated for $I_{\text {beam }}=315 \mathrm{~mA}$ by using formula (5). 


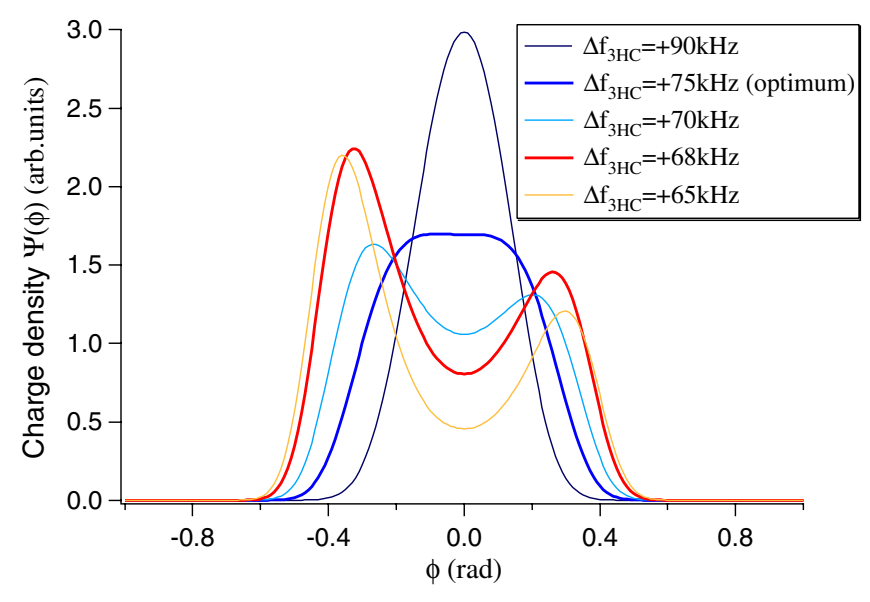

FIG. 15. (Color) Nominal charge density in the bunch in function of the $3 \mathrm{HC}$ detuning, calculated in uniform filling and at $315 \mathrm{~mA}$ by using formula (9).

best data fitting. Figure 16(c) corresponds to a detuning of $+64 \mathrm{kHz}$ and the bunch profile appears strongly overstretched, with two well-separated stable peaks. The experimental results in Fig. 16 are in very good agreement with the calculated bunch profile $\Psi(\phi)$ of formula (9), plotted in Fig. 15.

When a nonuniform filling is set, the phase shift along the bunch train does not allow one to average on all bunch profiles, but a separate analysis of the profile of each bunch needs to be performed. Entering in overstretching regime the bunch profile is affected by a combination of two effects: the phase shift and the splitting of the particles distribution in two peaks. The result is that the head and tail bunches have an anomalous stretched profile, with a single peak pulled on one side, as shown in Fig. 17. Otherwise the bunches in the middle, having a stable phase close to the center of the bucket, still assume a profile with two peaks [see Fig. 17(b)]. Enlarging the empty gap in the filling pattern amplifies this effect due to the increased phase shift. The next paragraph describes the observed beam lifetime improvement due to a low overstretching of the bunch profile, leading us to define a "low overstretching threshold." In fact, by increasing the harmonic voltage above the threshold, even if the bunch lengthening provides a positive increment in the Touschek lifetime, the latter decreases because of the progressive reduction of the bucket size (i.e., $r f$ acceptance).

\section{LIFETIME IMPROVEMENT}

In the absence of $3 \mathrm{HC}$ the longitudinal coupled-bunch instabilities dilute the bunches with a consequent beam lifetime increment with respect to the nominal value. Thus when we tune $3 \mathrm{HC}$, the measured beam lifetime is affected by the balancing between the damping of the longitudinal CBMs, which decreases the lifetime value, and the bunch lengthening that increases it. In order to study the lifetime improvement due to the $3 \mathrm{HC}$ activation and prevent it from being affected by instabilities contribution, a comparison has been carried out between the nominal beam lifetime and the lifetime value measured when the beam is completely stable and $3 \mathrm{HC}$ tuned at the operating point.

Before discussing the experiments on the beam lifetime improvement for different fillings, the authors would like to point out an experimental observation which has already been anticipated in the previous paragraphs. In all experiments at different fillings, $3 \mathrm{HC}$ was detuned beyond the optimum detuning, corresponding to the optimum harmonic voltage, and the measured beam lifetime increased even in the overstretching regime until a certain threshold. Assuming the bunches have a Gaussian longitudinal distribution, the beam lifetime is a function of the bunch length $\sigma_{s}$ [14], so it is increased when lengthening the bunch. If the Gaussian bunch profile is distorted, the key parameter to be considered is the rms bunch length, which has been obtained by the streak camera data. It has been observed that, when tuning $3 \mathrm{HC}$ just below the optimum
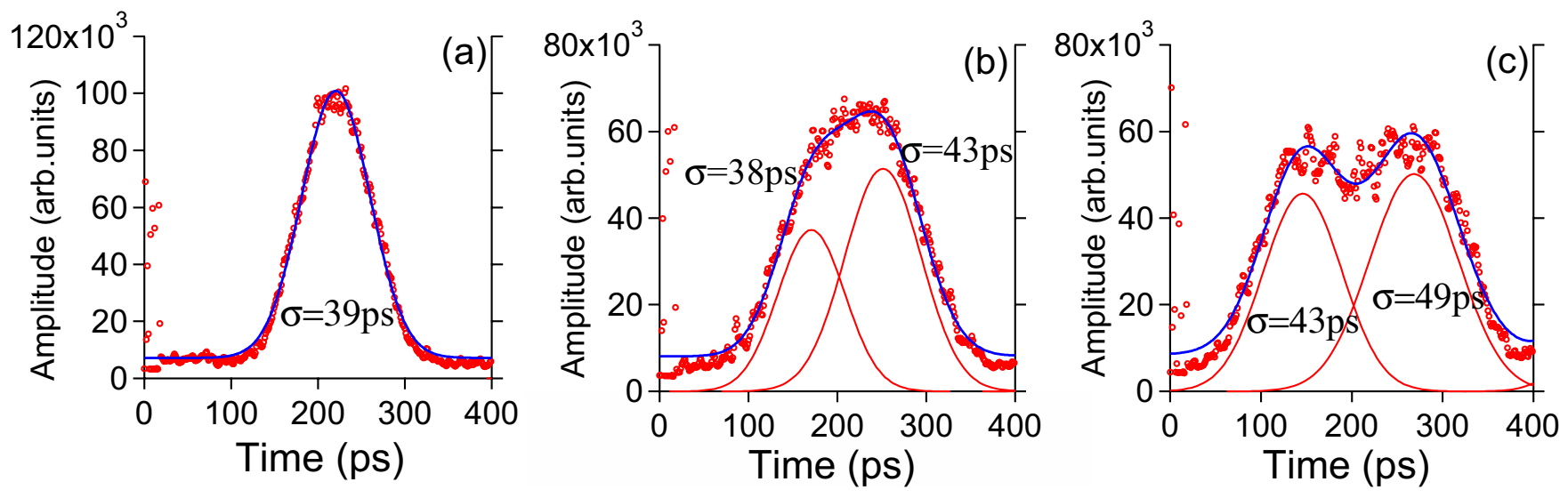

FIG. 16. (Color) Average bunch profile with the corresponding best fitting curves in case of a uniform filling for a $3 \mathrm{HC}$ detuning of $+114 \mathrm{kHz}$ (a), $+74 \mathrm{kHz}$, (b), and $+61 \mathrm{kHz}$ (c); $I_{\text {beam }}=315 \mathrm{~mA}, 2.0 \mathrm{GeV}$. 

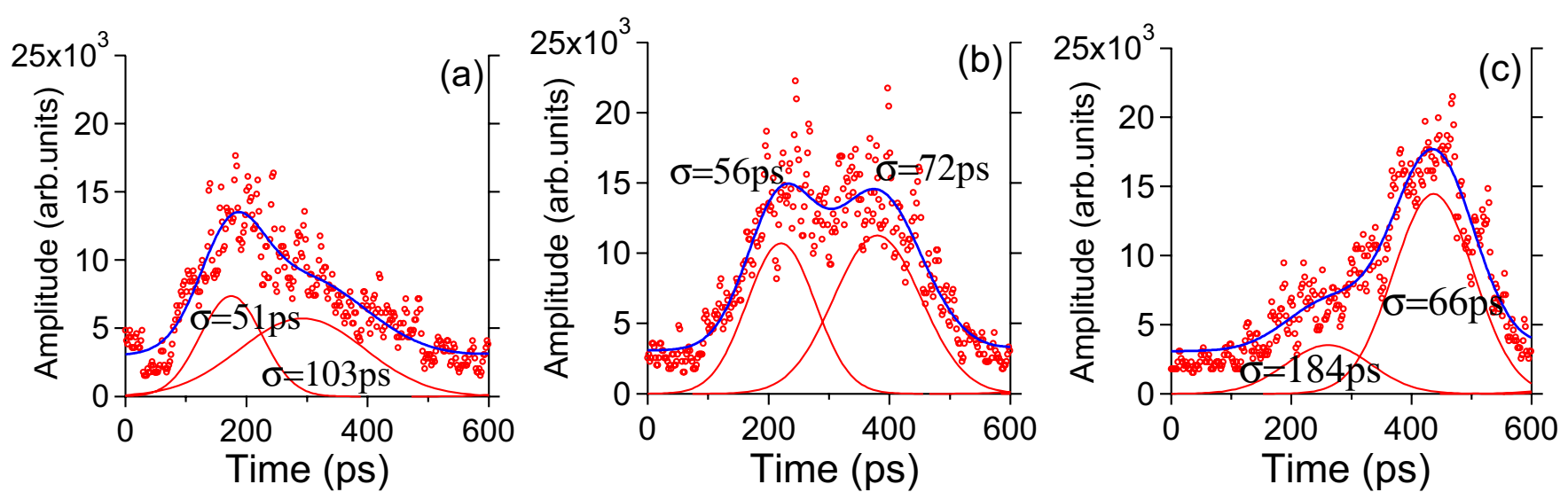

FIG. 17. (Color) Bunch profile in the tail (a), in the middle (b), and in the head (c) of the bunch train in case of a fractional filling of $96 \%, 3 \mathrm{HC}$ detuned of $+64 \mathrm{kHz} ; I_{\text {beam }}=315 \mathrm{~mA}, 2.0 \mathrm{GeV}$.

detuning, the distortion of the rf potential well and the corresponding reduction of the bucket size are well compensated by the large increasing of the rms longitudinal distribution. Hence the beam lifetime continues to increase even in a low overstretching regime, until it suddenly drops to a few hours when the longitudinal distribution exceeds a threshold and splits in two peaks.

This can be explained if we consider that, in order to calculate the improvement on the Touschek lifetime due to a harmonic system, inclusion of the effect of the rf acceptance reduction and of the modified density in the longitudinal distribution of particles $\Psi(\phi)$ needs to be performed. Then the Touschek lifetime $\tau_{T}$ may be expressed as [7]:

$$
\tau_{T, h}=\tau_{T, 0} \frac{\epsilon_{\mathrm{rf}, h}^{2}}{\epsilon_{\mathrm{rf}, 0}^{2}} \frac{\int \Psi_{0}^{2}(\phi) d \phi}{\int \Psi_{h}^{2}(\phi) d \phi}
$$

where the suffixes " $h$ " and " 0 " refer to the situation with and without the harmonic voltage. By using formula (10) we can calculate the Touschek lifetime increase factor $\tau_{T, h} / \tau_{T, 0}$ in function of the $3 \mathrm{HC}$ detuning for a beam current of $315 \mathrm{~mA}$. The results are plotted in Fig. 18.

The Touschek lifetime continues to increase up to the maximum value (an improvement factor of 3.78) that is in correspondence of a $3 \mathrm{HC}$ detuning of $+68 \mathrm{kHz}$. The optimum harmonic voltage calculated using formula (6) corresponds to a $3 \mathrm{HC}$ detuning of about $+75 \mathrm{kHz}$, and provides a factor 3.15 theoretical improvement: this confirms, as it has been observed, a little overstretching of the bunch profile improves the Touschek lifetime by an amount of $20 \%$ with respect to the optimum condition.

The conclusion is that, if a slight bunch overstretching is accepted, the optimum $3 \mathrm{HC}$ detuning from the point of view of the beam lifetime does not correspond to the optimum detuning previously defined as necessary to flatten the rf potential well.

Having found that the bunch lengthening is strongly affected by the empty gap size, the beam lifetime increment for several fractional fillings has been investigated.
Findings have shown that the best performance in terms of lifetime is obtained with a fractional filling of $96 \%$, (i.e., 415 filled buckets out of 432 available) that is now taken as the new standard filling pattern for Users Operation Mode. The uniform filling is not the optimum operational mode because of the presence of residual ions trapped in the ring, cleaned by that small "empty" gap $(\sim 4 \%)$. The beam lifetime measured at $2.0 \mathrm{GeV}$ and $320 \mathrm{~mA}$ currently reaches approximately $28 \mathrm{~h}$ (more than 3 times the value without $3 \mathrm{HC}$, see Table I): this large improvement has led to a modification of the routine operation, refilling the ring every $36 \mathrm{~h}$, instead of every 24 . Figure 19 shows the current decay in the ELETTRA storage ring with and without tuning $3 \mathrm{HC}$. In absence of $3 \mathrm{HC}$, after $24 \mathrm{~h}$ the beam current in the ring is about $100 \mathrm{~mA}$, while by tuning $3 \mathrm{HC}$ at the operating point after $36 \mathrm{~h}$ the accumulated current is still $115 \mathrm{~mA}$. Recently, the refilling rate has been decreased furthermore to $48 \mathrm{~h}$.

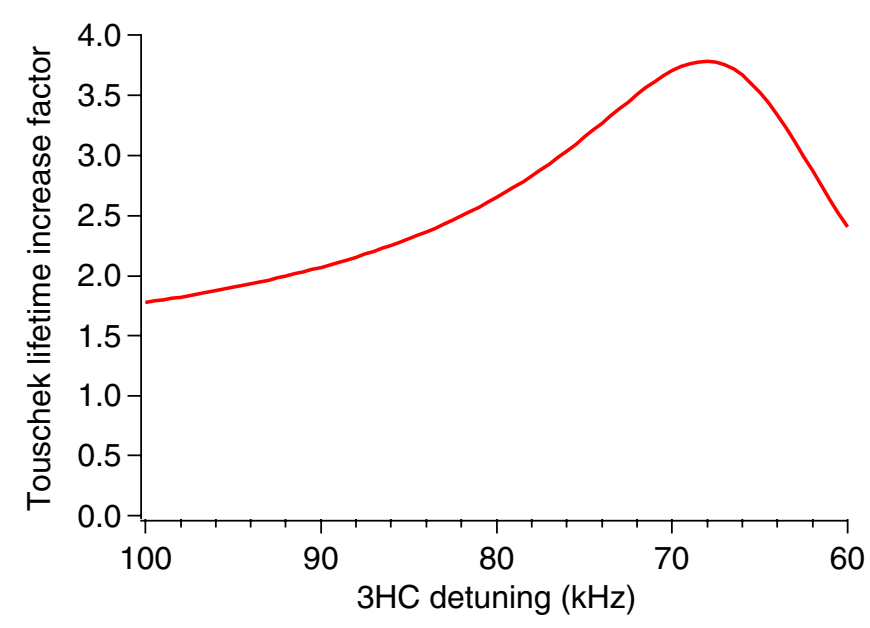

FIG. 18. (Color) Touschek lifetime increase factor in function of the $3 \mathrm{HC}$ detuning, calculated for a beam current of $315 \mathrm{~mA}$. 


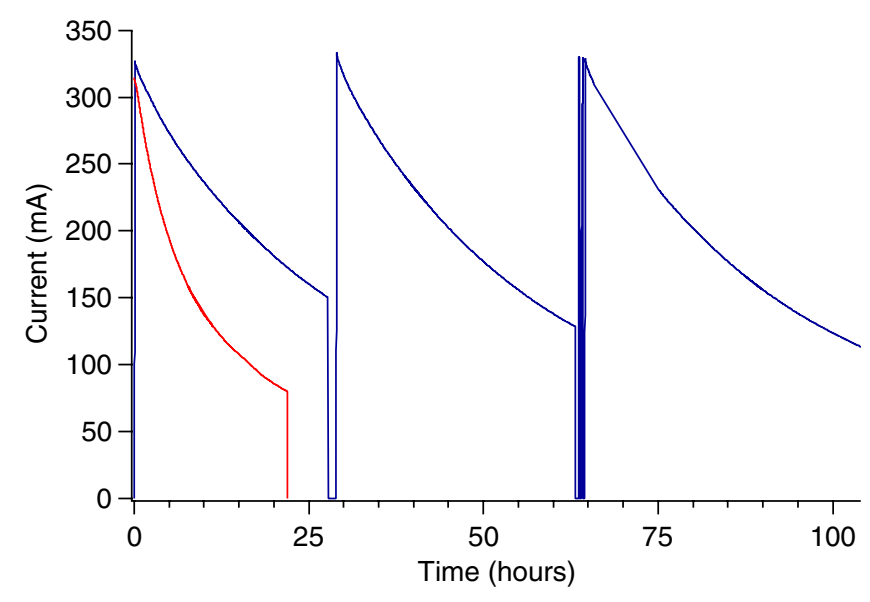

FIG. 19. (Color) Current decay comparison between ELETTRA operation with $3 \mathrm{HC}$ not active (red line) and with $3 \mathrm{HC}$ tuned (blue line). In this second case the interval between two subsequent injection is about $36 \mathrm{~h}$.

\section{BUNCH SHORTENING}

Even if the main reason installing a third harmonic cavity at ELETTRA lies in lengthening the bunch in order to improve the Touschek lifetime, for some specific experiments it could be interesting to operate the $3 \mathrm{HC}$ in bunch shortening mode by tuning it below the third harmonic of the beam. The harmonic voltage and the harmonic phase are adjusted to increase the total voltage slope seen by the beam, and the rf potential well around the synchronous phase is consequently modified. As in the case of the lengthening mode, the $3 \mathrm{HC}$ is operated in passive mode, so that the harmonic voltage generated by the beam passage depends on the frequency detuning, according to formula (3) described above, while the harmonic phase results to be very close to $-\pi / 2$. Under these conditions, the shortening factor with respect to the nominal $\mathrm{rms}$ bunch length at zero current ( $18.5 \mathrm{ps}$ ) is computed by using the formula (8) for different harmonic voltage, or similarly at different $3 \mathrm{HC}$ detuning.

In order to verify the real possibility to operate in shortening mode, $3 \mathrm{HC}$ has been tuned at approximately $-140 \mathrm{kHz}\left(f_{3 \mathrm{HC}}=1498.820 \mathrm{MHz}\right)$ and the storage ring has been operated with a uniform filling at $320 \mathrm{~mA}$, $2.0 \mathrm{GeV}$. This way, the beam proves to be longitudinally unstable, because the $3 \mathrm{HC}$ does not provide any Landau damping, so the streak camera equipment can not be used to measure the rms bunch length with acceptable precision. However, the increase of the voltage slope around the synchronous phase allows for considering the synchrotron frequency as a reliable parameter to calculate the rms bunch length, which in linear approximation is inversely proportional to synchrotron frequency. Thus $3 \mathrm{HC}$ has been progressively tuned down to $-60 \mathrm{kHz}$, which is the minimum detuning reached before losing the beam, and the synchrotron frequency has been measured at each step: the average shortening factor is obtained by comparing these

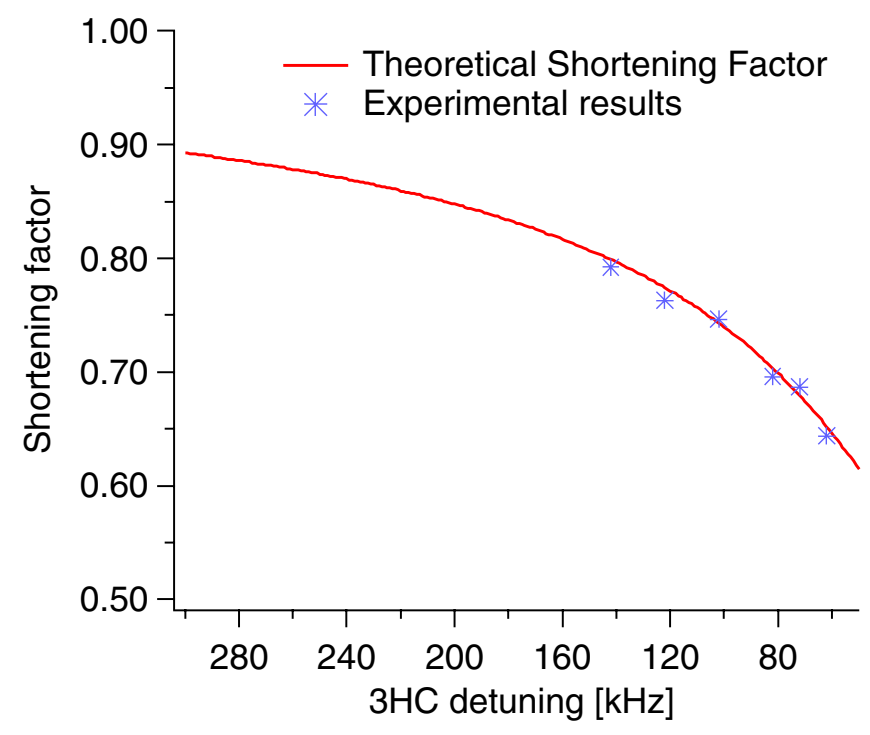

FIG. 20. (Color) Theoretical bunch shortening factor in function of the $3 \mathrm{HC}$ detuning.

values with the synchrotron frequency measured with $3 \mathrm{HC}$ parked ( $\sim 10.3 \mathrm{kHz})$. Figure 20 reveals a very good correspondence between our experimental results and the theoretical bunch shortening curve.

We can finally conclude that $3 \mathrm{HC}$ can be operated in shortening mode at $320 \mathrm{~mA}, 2.0 \mathrm{GeV}$ reducing the rms bunch length by a factor 1.6.

\section{CONCLUSION}

The installation of the superconducting third harmonic cavity in the ELETTRA storage ring has improved the beam lifetime by more than a factor of 3 with respect to the nominal beam lifetime of ELETTRA. An in-depth investigation of the filling pattern influence on the benefit provided by the $3 \mathrm{HC}$ activation has been carried out. The phase shift along the bunch train, induced by the empty gap, significantly reduces the $3 \mathrm{HC}$ effectiveness, especially concerning the beam lifetime improvement. Measuring the beam lifetime at several $3 \mathrm{HC}$ detunings shows a lifetime increase even beyond the optimum detuning, corresponding to the optimum harmonic voltage necessary to completely flatten the rf potential. This means that the bunches must be slightly overstretched and the fact that, in the uniform filling case, a "low overstretching threshold" exists, for which the Touschek lifetime increase is maximum, has been demonstrated. When exceeding this threshold, the lifetime abruptly drops. This threshold has been measured not only for the uniform filling case, finding a very good correspondence with the calculation, but also for fractional fillings. Moreover, the performance of $3 \mathrm{HC}$ has been compared, in terms of beam lifetime, for several fractional filling patterns, showing that best conditions are met in the case of a $96 \%$ filling. This fact suggests that, even if the maximum bunch lengthening is reached with 
uniform filling, ELETTRA requires a small empty gap to optimize the beam lifetime, in order to clean the residual trapped ions.

The superconducting third harmonic cavity has been in routine operation at ELETTRA since September 2003, and the new standard User's operation envisages a filling pattern of $96 \%$. The great improvement in beam lifetime allows for refilling of the ring every $48 \mathrm{~h}$, instead of every 24 , which is of greatest importance for reliable and stable operation for the Users'. Moreover, the slower current decay provides a relevant benefit even for the machine thermal stability.

\section{ACKNOWLEDGMENTS}

The authors would like to thank V. Serrière and J. Jacob for the very useful discussions concerning the understanding of the beam dynamics, and for providing access to their multiparticles tracking code. They are also grateful to $\mathrm{M}$. Ferianis and M. Trovò for their assistance in the use of the Streak Camera equipment and in the elaboration of the acquired data. Exceptional acknowledgements go to the ELETTRA machine operators group for the incredibly valuable support during the measurements of the $3 \mathrm{HC}$ performance in the physics machine studies shifts, and to the group leader E. Karantzoulis for his support in writing this paper.

[1] M. Svandrlik, C. J. Bocchetta, A. Fabris, F. Iazzourene, E. Karantzoulis, R. Nagaoka, C. Pasotti, L. Tosi, R. P. Walker, and A. Wrulich, in Proceedings of the 1995 Particle Accelerator Conference, Dallas, TX, 1995 (IEEE, Piscataway, NJ, 1995), pp. 2762-2764.

[2] G. Penco, Ph.D. thesis, Sincrotone Trieste-University of Milan, 2004.
[3] T. Furuya, K. Akai, K. Asano, E. Ezura, K. Hara, K. Hosoyama, A. Kabe, Y. Kojima, S. Mitsunobu, Y. Morita, H. Nakanishi, T. Shishido, T. Tajima, T. Takahashi, T. Takashima, S. Yoshimoto, Y. Ishi, Y. Kijima, T. Murai, and K. Sennyu, in Proceedings of the 1996 European Particle Accelerator Conference, Sitges, Spain (IOP, Bristol, 1996), pp. 2121-2123.

[4] A. Mosnier, S. Chel, X. Hanus, A. Novokhatski, and G. Flynn, in Proceedings of the Particle Accelerator Conference, Vancouver, BC, Canada, 1997 (IEEE, Piscataway, NJ, 1997), pp. 1709-1711.

[5] P. Marchand, in Proceedings of the 1999 Particle Accelerator Conference, New York, 1999 (IEEE, Piscataway, NJ, 1999), pp. 989-991.

[6] J. M. Byrd, K. Baptiste, S. De Santis, S. Kosta, C. C. Lo, D. Plate, R. A. Rimmer, and M. Franks, Nucl. Instrum. Methods Phys. Res. 439, 15 (2000).

[7] J. M. Byrd and M. Georgsson, Phys. Rev. ST Accel. Beams 4, 030701 (2001).

[8] A. Hofmann and S. Myers, in Proceedings of the 11th International Conference on High Energy Accelerators (CERN Birkhaser Verlag, Basel, 1980); Report No. ISRTH-RF/80-26, 1980.

[9] J. M. Byrd, S. De Santis, J. Jacob, and V. Serrière, Phys. Rev. ST Accel. Beams 5, 092001 (2002).

[10] V. Serrière, Ph.D. thesis, University Paris XI, 2002.

[11] O. Naumann, Ph.D. thesis, University of Berlin, 1999.

[12] M. Ferianis, in Proceedings of the 4th European Workshop on Diagnostic and Instrumentation for Particle Accelerators, DIPAC99, Chester, UK, 1999 (CLRC Daresbury Laboratory, Daresbury, United Kingdom, 1999).

[13] M. Ferianis and M. Danailov, in Beam Instrumentation Workshop 2002: Tenth Workshop, edited by G. A. Smith and T. Russo, AIP Conf. Proc. No. 648 (AIP, New York, 2002).

[14] J. Le Duff, CERN Report No. CERN 98-06, 1998. 